\title{
Emotion Classification of Restaurant and Laptop Review Dataset: Semeval 2014 Task 4
}

\author{
D. K. Kirange \\ Dept. of Computer and IT, J T Mahajan College of \\ Engineering, \\ Faizpur, Tal, Yawal, Dist. Jalgaon, India
}

\author{
Ratnadeep R. Deshmukh, Ph.D. \\ Dept of Computer Science and IT \\ Dr. Babasaheb Ambedkar Marathwada University, \\ Aurangabaad , India
}

\begin{abstract}
The "Sentiment Analysis" task focuses on the recognition and classification of emotions (positive, negative, conflict, neutral) in reviews for the aspect. In this paper we propose the system for recognizing and analyzing the sentiments using SVM for the restaurant and laptop review dataset. We compare the performance of the system with well-known KNN classifier.
\end{abstract}

\section{Index Terms}

Aspect, Sentiment Analysis, SVM, KNN

\section{INTRODUCTION}

Sentiment analysis (or opinion mining) is defined as the task of finding the opinions of authors about specific entities. The decision-making process of people is affected by the opinions formed by thought leaders and ordinary people. When a person wants to buy a product online he or she will typically start by searching for reviews and opinions written by other people on the various offerings. Sentiment analysis is one of the hottest research areas in computer science. A sentiment analysis model is used to analyze a text string and classify it with one of the labels that you provide; for example, you could analyze a tweet to determine whether it is positive or negative, or analyze an email to determine whether it is happy, frustrated, or sad. Aspect-based sentiment analysis is the research problem that focuses on the recognition of all sentiment expressions within a given document and the aspects to which they refer.

It is common to classify sentences into two principal classes with regard to subjectivity: objective sentences that contain factual information and subjective sentences that contain explicit opinions, beliefs, and views about specific entities. Here, I mostly focus on analyzing subjective sentences. As an example, here is a review about a hotel in Manhattan.

"The king suite was spacious, clean, and well appointed. The reception staff, bellmen, and housekeeping were very helpful. Requests for extras from the maid were always provided. The heating and air conditioning functioned well; this was good as the weather was variable. The sofa bed was the best I've ever experienced. The king size bed was very comfortable. The building and rooms are very well soundproofed. The neighborhood is the best for shopping, restaurants, and access to subway. Only "complaint" has to do with high-speed Internet access. It's only available on floors $8-12 . "$

Overall the review is very positive about the hotel. It refers to many different aspects of the hotel including: heating, air conditioning, staff courtesy, bed, neighborhood, and Internet access. In this paper we propose sentiment analysis systems which are able to provide a sentiment score for the whole review as well as analyze the sentiment of each individual aspect of the hotel.

In this paper we propose a sentiment analysis task of SemEval 2014 [6] which deals with the laptops review dataset. Particularly we have considered the three subtasks

- Identification of sentiment term which is usually present in the sentence,

- recognition of sentiment category and

- Finally sentiment polarity detection for the sentiment category.

We have used dictionary of positive and negative keywords from the dictionary if LIWC [7] and SVM for classification.

\section{RELATED WORK}

In [1] , sentiment classification techniques are incorporated into the domain of political news from columns in different Turkish news sites. Authors have compared four supervised machine learning algorithms of Naïve Bayes, Maximum Entropy, SVM and the character based N-Gram Language Model for sentiment classification of Turkish political columns. We Also the problem of sentiment classification in the political news domain is discussed. It is observed from empirical findings that the Maximum Entropy and N-Gram Language Model outperformed the SVM and Naïve Bayes. Using different features, all the approaches reached accuracies of $65 \%$ to $77 \%$.

A generative probabilistic topic model is proposed in [2] that detects both an aspect and corresponding sentiment, simultaneously, from review articles. Unlike existing sentiment analysis models, which generally consider rating prediction to be a side task, the proposal [2] , the hierarchical approach to sentiment analysis, identifies both an item and its rating by dividing topics, traditionally treated as one entity, into aspect and sentiment topics. Since the model is aware of both objective and subjective information, it can discover finegrained tightly coherent topics, and describe the generative process of each article in a unified manner.

A Portuguese dictionary focused in a specific field of study was built in [3], in which tenses and negative words are treated in a different way to measure the polarity, the strength of positive or negative sentiment, in short texts extracted from Twitter. For the Portuguese Dictionary performance validation, the results are compared with the SentiStrength tool and are evaluated by three Specialists in the field of study; each one analyzed 2000 texts captured from Twitter. Comparing the efficiency of the SentiMeter-Br and the SentiStrength against the Specialists' opinion, a Pearson correlation factor of 0.89 and 0.75 was reached, respectively, proving that the metric used in the Sentimeter- $\mathrm{Br}$ is better 
than the one used in the SentiStrength. The polarity of the short texts were also tested through machine learning, with correctly classified instances of $71.79 \%$ by Sequential Minimal Optimization algorithm and F-Measure of 0.87 for positive and 0.91 for negative phrases.

In [4] a method to handel sentiment Analysis is proposed for Cantonese opinion mining. In [4] authors use Hidden markov model (HMM) to conduct word segmentation, and then building opinion orientation dictionary for Cantonese. Also a method for feature orientation summarization is proposed. It is the first work of sentiment analysis for Cantonese opinion mining.

The study in [5] aims to look beyond the quantitative summary to provide a more comprehensive view of online user-generated content. Authors obtain a unique and extensive dataset of online user reviews for hotels across various review sites and over a long time periods. Authors in [4] use the sentiment analysis technique to decompose user reviews into five dimensions to measure hotel service quality. Those dimensions are then incorporated into econometrics models to examine their effect in shaping users' overall evaluation and content generating behavior.

\section{TASK DEFINITION}

Sentiment analysis (also known as opinion mining) refers to the use of natural language processing, text analysis and computational linguistics to identify and extract subjective information in source materials. A basic task in sentiment analysis is classifying the polarity of a given text at the document, sentence, or feature/aspect level - whether the expressed opinion in a document, a sentence or an entity feature/aspect is positive, negative, or neutral. Sentiment analysis is increasingly viewed as a vital task both from an academic and a commercial standpoint. The majority of current approaches, however attempt to detect the overall polarity of a sentence, paragraph or text span, regardless of the entities mentioned (e.g. restaurants, laptops) and their aspects (e.g food, price, service). In contrast this task focuses on sentiment analysis where the goal is to identify aspect of the given target entity and sentiment expressed in each review.

In particular, the paper focuses on three tasks of SemEval 2014 Conference [6].

\subsection{Subtask 1: Sentiment Term Extraction:}

Given a set of sentences with pre-defined entities (e.g. laptops), identify the sentiment terms present in the sentence and return a list containing all the distinct sentiment terms.

For example, "I liked the battery and the display, but not the sound,

\subsection{Subtask 2: Sentiment Term Polarity}

For a given set of sentiment terms within a sentence determine whether the polarity of each sentiment term is positive, negative, neutral or conflict.

For example, -I loved their Products

\subsection{Laptops Reviews Dataset}

This dataset provided as a train and trial dataset in [6], consists of over $3 \mathrm{~K}$ English sentences from the laptop reviews.
The sentences in the datasets are annotated using XML tags.

The following example illustrates the format of the annotated sentences of the laptops dataset. The format is the same as in the laptop datasets, with the only exception that there are no annotations for aspect categories. Notice that we annotate only aspect terms naming particular aspects (e.g., "everything about it" does not name a particular aspect).

<sentence id="353">

<text>From the build quality to the performance, everything about it has been sub-par from what I would have expected from Apple. $</$ text $>$

<aspectTerms>

<aspectTerm term="build quality"

polarity="negative" from="9" to="22"/>

<aspectTerm term="performance"

polarity="negative" from="30" to="41"/>

$</$ aspectTerms $>$

$</$ sentence $>$

\subsection{Restaurant Reviews Dataset}

This dataset provided as a trial dataset in [6], consists of over $3 \mathrm{~K}$ English sentences from the restaurant reviews.

The sentences in the data sets are annotated using XML tags.

The following example illustrates the format of the annotated sentences of the restaurants dataset.

<sentence id="813">

$<$ text $>$ All the appetizers and salads were fabulous, the steak was mouth watering and the pasta was delicious!! $!</$ text $>$

<aspectTerms>

<aspectTerm term="appetizers"

polarity="positive"/>

$<$ aspectTerm term="salads"

polarity="positive"/>

<aspectTerm term="steak" polarity="positive"/>

<aspectTerm term="pasta" polarity="positive"/>

$</$ aspectTerms $>$

<aspectCategories>

$<$ aspectCategory category="food"

polarity="positive"/>

$</$ aspectCategories $></$ sentence $>$

The possible values of the polarity field are: -positivell, -negativell, - conflictl, -neutrall. The possible values of the category field are: -foodll, - servicell, - pricell, —ambiencell, —anecdotes/miscellaneousll.

\section{SENTIMENT ANALYSIS}

The task focuses on identification of sentiment term from the sentence, then accordingly identifying polarity for the laptop review sentence. We have used the dictionary based approach for recognizing the sentiment terms present in the sentence. To analyze the sentiment of the sentence we have used SVM classifier[7].The sentiments are classified as positive, negative, conflict, neutral etc. 


\subsection{Sentiment Term Extraction}

We have used a dictionary based approach for recognizing the occurrence of a particular sentiment term in the sentence..

\subsection{Aspect Term Polarity}

For determining the sentence polarity regarding the aspect term, we have used the SVM [8] classifier. We have classified the sentiments as positive, negative, conflict and neutral.

- We have used the dictionary of positive and negative keywords i.e. dictionary of LIWC [9].

- For each sentence in the laptop review, we have prepared a term-document training matrix showing the occurrence of the positive or negative term.

- We have trained our SVM classifier with this matrix and tested the same with some test data.

- The sentences which contain both positive and negative keywords are treated as conflicts while the sentences which do not contain any keywords (positive or negative) are treated as neutral.

\section{PERFORMANCE EVALUATION}

We have identified the various sentiment categories as positive, negative, neutral and conflict etc. The well known SVM classifier is used for classification of a review sentence in appropriate category. The result is compared with the standard annotations provided in the dataset and the accuracy is computed as

$$
\text { Accuracy }=\text { sum }(\text { abs }(\text { Expected output }- \text { actual output })) / 2
$$

The classification results are compared with the $\mathrm{KNN}$ classifier as shown in the table 1 .

The dictionary of LIWC is used for positive and negative keywords. The SVM [10] classifier is trained with the term document matrix prepared using the LIWC dictionary of keywords. The result is compared with the standard annotations provided and the accuracy is computed as above. We have tested the performance of SVM classification with $\mathrm{KNN}$ as shown in table 1 and 2 .

Table 1. Accuracy for Laptop Dataset

\begin{tabular}{|l|c|c|}
\hline \multicolumn{1}{|c|}{$\begin{array}{c}\text { Sentiment } \\
\text { Term } \\
\text { Polarity }\end{array}$} & \multicolumn{2}{|c|}{ Accuracy } \\
\hline & SVM & KNN \\
\hline Positive & 62.7273 & 60.00 \\
\hline Negative & 63.6364 & 49.29 \\
\hline Neutral & 78.1818 & 24.5098 \\
\hline Conflict & 97.2727 & 58.16 \\
\hline Average Accuracy & 75.4545 & 47.9899 \\
\hline
\end{tabular}

Table 2. Accuracy for Restaurant Dataset

\begin{tabular}{|l|l|c|}
\hline \multicolumn{1}{|c|}{$\begin{array}{c}\text { Sentiment Term } \\
\text { Polarity }\end{array}$} & \multicolumn{2}{|c|}{ Accuracy } \\
\hline & 71.9212 & 46.58 \\
\hline Positive & 100 & 81.7734 \\
\hline Negative & 90.6404 & 71.0660 \\
\hline Neutral & 99.5074 & 69.13 \\
\hline Conflict & 90.5172 & 67.1373 \\
\hline Average Accuracy & & \\
\hline
\end{tabular}

\section{CONCLUSIONS}

In the paper, we propose a method to handle sentiment analysis task of SemEval 2014 workshop for Restaurant and Laptop review dataset. In our methods, we use Support Vector Machines (SVM) for recognizing sentiment categories and sentiment polarity. The sentiment polarities are classified as positive, negative, neutral and conflict etc. The classification results are compared with the ground truth annotations provided with the dataset and the result is compared with the performance of KNN classifier.

\section{ACKNOWLEDGMENT}

The authors would like to welcome the reviewers for their critical and constructive comments and suggestions. The authors would like to thank the Department of Computer Science of Dr. Babasaheb Ambedkar Marathwada University, Aurangabaad for providing the infrastructure to carry out the research.

\section{REFERENCES}

[1] Mesut Kaya, Guven Fidan, Ismail H. Toroslu1J., - Sentiment Analysis of Turkish Political Newsll, 9780-7695-4880-7/12, 2012 IEEE Computer Society

[2] Noriaki Kawamae, -Hierarchical Approach to Sentiment Analysis\|, 2012 IEEE Sixth International Conference on Semantic Computing.

[3] Renata Lopes Rosa, Demostenes Zegarra Rodriguez, Graca Bressan, - SentiMeter-Br: a Social Web Analysis Tool to Discover Consumers' Sentimentl, 2013 IEEE 14th International Conference on Mobile Data Management.

[4] Jian Chen, Yu Liu, Guangyi Zhang, - Sentiment Analysis for Cantonese Opinion Mining\|, 2013 Fourth International Conference on Emerging Intelligent Data and Web Technologies.

[5] Wenjing Duan, Qing Cao, Yang Yu, Stuart Levy, -Mining Online UserGenerated Content: Using Sentiment Analysis Technique to Study Hotel Service Qualityll, 2013 46th Hawaii International Conference on System Sciences, 1530-1605/12 $2012 \quad$ IEEE http://alt.qcri.org/semeval2014/task4/

[6] D K Kirange, Dr, R R Deshmukh, "Emotion Classification of news headlines using SVM" Asian 
International Journal of Computer Applications (0975 - 8887)

Volume 113-No. 6, March 2015

Journal of Computer Science \& Information Technology $2(5)$

[7] Dr. Deshmukh R. R. , Kirange D. K., "Classifying News Headlines for Providing User Centered E-Newspaper using SVM", International Journal of Emerging Trends in Technology and Computer Science (IJETTCS) June $2013 \mathrm{http} / / / \mathrm{www}$. liwc.net

[8] Kirange D. K., Dr. Deshmukh R. R., "Aspect Based Sentiment Analysis: Semeval 2014- Task-4", Asian
Journal of Computer Science and Information Technology (AJCSIT) Vol 4, No 8 (2014)

\section{AUTHOR'S PROFILE}

D. K Kirange, Research Student in Department of Computer Sci. and Information Technology, Dr. Babasaheb Ambedkar Marathwada University, Aurangabad, M.S., India.

R. R. Deshmukh, Professor in Department of Computer Sci. and Information Technology, Dr. Babasaheb Ambedkar Marathwada University, Aurangabad, M.S., India 